\title{
Baseline levels of serum high sensitivity $C$ reactive protein and lipids in predicting the residual risk of cardiovascular events in Chinese population with stable coronary artery disease: a prospective cohort study
}

Wen Dai ${ }^{\dagger}$, Ziyu Zhang ${ }^{\dagger}$ and Shuiping Zhao ${ }^{*}$

\begin{abstract}
Background: The contributions of inflammation, triglyceride (TG) and high-density lipoprotein cholesterol (HDL-C) to the residual risk of cardiovascular events have not been determined in a large cohort of Chinese population before. This study was aimed to investigate the association of serum levels of high sensitive $C$ reactive protein (hs-CRP), TG and $\mathrm{HDL}-\mathrm{C}$ with the residual risk of cardiovascular events in patients with stable coronary artery disease (CAD).

Methods: We enrolled 4090 patients with stable CAD from 13 hospitals in China. All participants received optimal medical treatment (OMT) for stable CAD suggested by guidelines and were followed. The endpoint measures were the first occurrence of a major adverse cardiovascular event (MACE), defined as cardiovascular death, non-fatal myocardial infarction, non-fatal stroke or unplanned coronary revascularization. Cox proportional regression analysis was conducted to identify independent predictors of MACE.

Results: We found that hs-CRP and HDL-C levels were associated with coronary lesion severity at baseline (both $p<0.001$ ). After 3 months OMT, 91.2\% (3730/4090) patients achieved the therapeutic goal for low density lipoprotein cholesterol (LDL-C) (<1.8 mmoL/L). During a mean follow-up period of 39.5 months, 11.5\% (471/4090) patients suffered MACE. In multivariate Cox proportional regression analysis, the hazard ratio for MACE was 1.17 (95\% confidence interval: $1.07-1.28, p<0.001)$ per standardized deviation in the log-transformed hs-CRP levels after adjustment for other traditional cardiovascular risk factors. However, baseline TG and HDL-C levels were not associated with MACE in this study.
\end{abstract}

Conclusions: Baseline hs-CRP level was an independent predictor of residual risk of cardiovascular events in Chinese population with stable CAD. However, TG and HDL-C levels were not associated with MACE.

Keywords: Coronary artery disease, Inflammation, High sensitivity-C reactive protein, Triglyceride, High density lipoprotein cholesterol

\footnotetext{
* Correspondence: xyzhaosp@sina.com

${ }^{+}$Wen Dai and Ziyu Zhang contributed equally to this work.

Department of Cardiology, The Second Xiangya Hospital, Central South

University, No. 139, Middle Renmin Road, Changsha 410011, China
}

(c) The Author(s). 2018 Open Access This article is distributed under the terms of the Creative Commons Attribution 4.0 International License (http://creativecommons.org/licenses/by/4.0/), which permits unrestricted use, distribution, and reproduction in any medium, provided you give appropriate credit to the original author(s) and the source, provide a link to the Creative Commons license, and indicate if changes were made. The Creative Commons Public Domain Dedication waiver (http://creativecommons.org/publicdomain/zero/1.0/) applies to the data made available in this article, unless otherwise stated. 


\section{Background}

Low density lipoprotein cholesterol (LDL-C) is a well-established pathogenic risk factor of coronary artery disease (CAD) [1, 2]. However, residual cardiovascular risk in population is still remained in the setting of controlled plasma LDL-C level and thus has been a major concern $[3,4]$. Therefore, efforts to identify other modifiable risk factors to further reduce residual cardiovascular risk are needed.

Inflammation has long been regarded as a critical participant in CAD development. More importantly, the recent trial has demonstrated that treatment with canakinumab targeting interleukin-1 $\beta$ (IL- $\beta$ ), an important component of immune reaction, significantly reduced cardiovascular event rate [5]. Thus, anti-inflammation therapy emerges as a novel intriguing approach to deal with CAD in clinical practice.

The role of high-density lipoprotein cholesterol (HDL-C) and triglycerides (TGs) in CAD still remain controversial. Although observational studies found that HDL-C and TG levels are both closely associated with the risk of CAD $[6,7]$, randomized controlled trials have failed to show that medications designed to increase HDL-C or decrease TG levels have any significant clinical benefits [8-13]. Interestingly, several large-scale genetic studies have consistently shown that some variants in gene loci involved in serum TG metabolism are associated with the risk of CAD [14-16], leading to the renewed interests of researchers on unraveling the role of TG in CAD.
However, the contribution of inflammation, TG and HDL-C to the residual risk of cardiovascular events have not been determined in a large cohort of Chinese population before. Cardiovascular risk attributed to inflammation and those serum lipid parameters may vary among different ethnics due to the differences in genetic background and life style.

Therefore, we investigated the values of serum high sensitivity $C$ reactive protein (hs-CRP), a routine inflammatory biomarker for cardiovascular risk assessment, TG and HDL-C in predicting major adverse cardiovascular event (MACE) in a cohort of Chinese population with stable CAD who received optimal medical treatment (OMT).

\section{Methods}

Study design and population

This is a prospective cohort study. Initially, we recruited patients with stable CAD from 13 hospitals in China from February 2013 to December 2013. Their baseline characteristics were recorded. Afterwards, all participants were followed by study investigators and the data of MACE were collected. Statistical analyses were conducted to investigate the association between baseline levels of hs-CRP, TG and HDL-C and MACE. The selection process of study population was illustrated in Fig. 1.

As described in previous study [17], patients were diagnosed with stable CAD if they presented with one of the following clinical phenotypes as a result of significant coronary artery atherosclerotic stenosis: 1) stable angina:

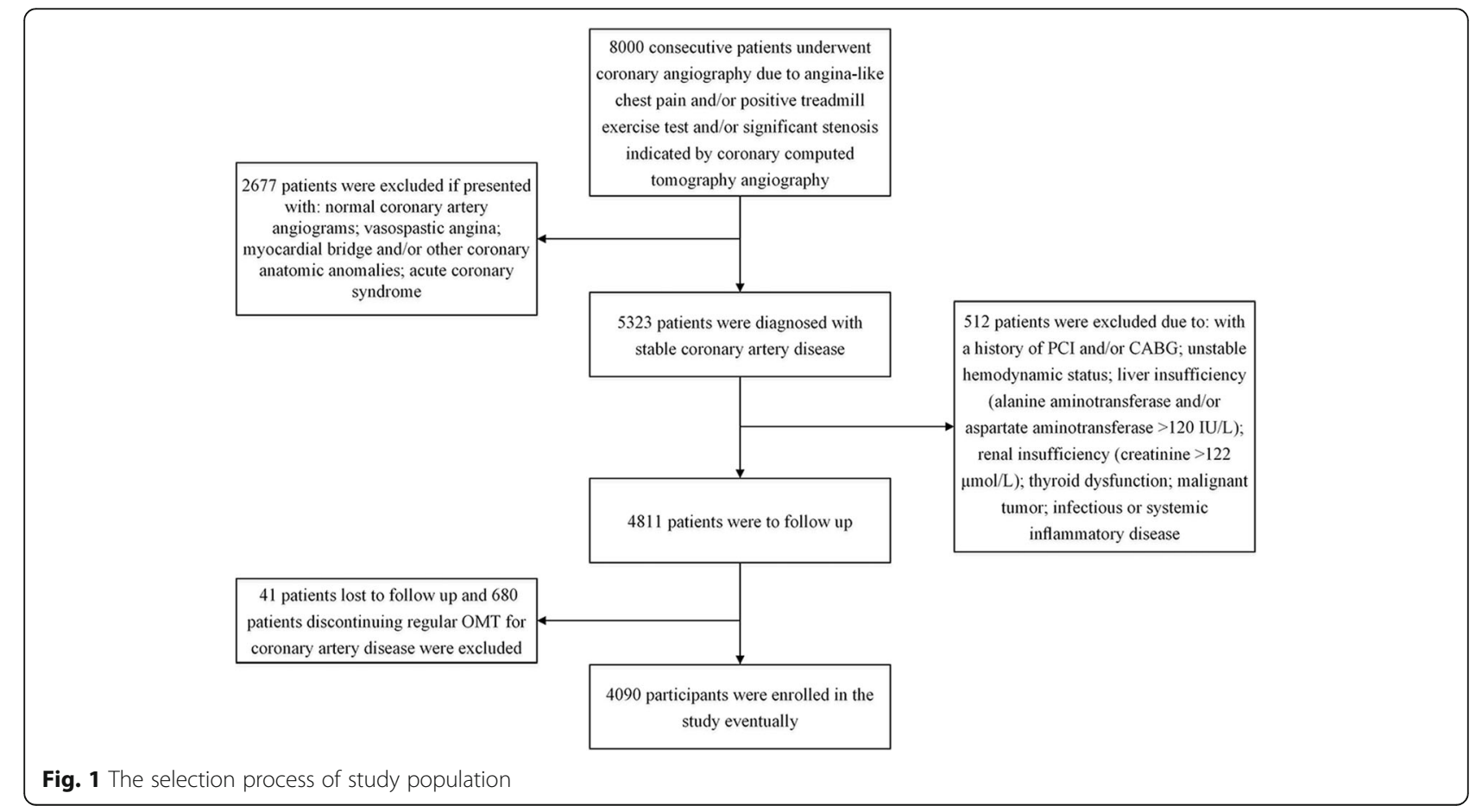


chest pain precipitated by physical activity that remits with rest; 2) ischemic cardiomyopathy: cardiomyopathy caused by the atherosclerotic narrowing of coronary arteries; 3) latent coronary artery disease: disease characterized by myocardial ischemia and coronary stenosis that are identifiable by medical tests but not with apparent clinical symptoms $[18,19]$. Contrarily, acute coronary syndrome (including unstable angina and myocardial infarction), vasospastic angina, and microvascular angina were not considered into the scope of stable CAD. Patients were excluded from the study for the criteria as follows: with myocardial bridge and/or other coronary anatomic anomalies; history of percutaneous coronary intervention (PCI) and/or coronary artery bypass grafting (CABG); unstable hemodynamic status; renal insufficiency (creatinine > $122 \mu \mathrm{mol} / \mathrm{L}$ ); liver insufficiency (alanine aminotransferase and/or aspartate aminotransferase >120 IU/L); thyroid dysfunction; infectious or systemic inflammatory disease; malignant tumor; discontinue regular OMT for stable CAD during follow-up; lost to follow-up.

This study was approved by the ethics committee review board of The Second Xiangya Hospital of Central South University. The study was carried out in accordance with the 1975 Declaration of Helsinki and the relevant regulations. Informed written consent was obtained from all participants.

\section{Data collection}

Demographic characteristics, serum levels of lipids and hs-CRP, and coronary atherosclerosis severity at baseline were recorded. Data of MACE of participants were collected during follow-up period. We also measured the serum levels of lipids and hs-CRP at the end of the 12-week after recruitment. The following events were considered MACE: 1) cardiovascular deaths: deaths attributable to cardiovascular causes; 2) non-fatal myocardial infarctions: myocardial infarctions that did not result in death; 3) non-fatal strokes: strokes that did not result in death; and 4) unplanned coronary revascularizations: unscheduled PCI or CABG. The study investigators obtained follow-up information at regular intervals via face-to-face or telephone interviews. The follow-up period lasted from the time of recruitment to January 2017 or the date of a MACE.

All participants were prescribed with OMT for stable CAD, suggested by recent guidelines [1], during the follow-up period as follows: 1) antithrombotic agents: aspirin and/or clopidogrel; 2) anti-ischemic agents: nitrates and/or beta-receptor blockers ( $\beta$-blockers) and/or calcium channel-blocking (CCB) agents; 3 ) renin-angiotensin inhibitors; and 4) LDL-C-lowering agents: statins.

As described in our previous study [17], blood samples were drawn by venipuncture after at least $10 \mathrm{~h}$ of overnight fasting. The blood specimens were processed and assessed at the central laboratory in each hospital. All clinical laboratories included in this study were standardized and certified. An automatic biochemistry analyzer (Hitachi 7360; Hitachi Ltd., Tokyo, Japan) and commercially available agents were used to measure serum total cholesterol (TC), LDL-C, HDL-C, TG, hs-CRP, fasting glucose and Hemoglobin A1c (HbA1c) levels. Hs-CRP and HbA1c levels were measured via turbidimetric immunoassay, and TC, LDL-C, HDL-C, TG, and glucose levels were measured using enzymatic assay. The left ventricular ejection fraction (LVEF) was determined by cardiac ultrasound examination. Coronary angiographic data were collected from patient catheterization laboratory records by at least 3 interventional cardiologists. Coronary lesion severity was assessed in each patient by the Gensini score (GS) [20], which was calculated by scoring each atherosclerotic lesion according to the degree of coronary artery luminal narrowing and the location of the lesion. The total score was calculated as a sum of the product of the stenosis and location score of each affected lesion.

The traditional risk factors for CAD were defined as described in our previous studies [17, 21]. Hypertension was defined as blood pressure $\geq 140 / 90 \mathrm{mmHg}$ in more than two measurements and/or the requirement of treatment with anti-hypertension drugs. Diabetes mellitus was defined as fasting serum glucose levels $\geq 7.0 \mathrm{mmol} / \mathrm{L}$, and/or random serum glucose $\geq 11.1 \mathrm{mmol} / \mathrm{L}$, and/or 2 -h post-prandial serum glucose $\geq 11.1 \mathrm{mmol} / \mathrm{L}$ on the oral glucose tolerance test in multiple determination and/or the requirement of treatment with hypoglycemic agents. The BMI was calculated as weight divided by height squared. Current smokers were subjects who had smoked regularly within the previous 12 months.

All study investigators underwent a training program and fully understood the aims of the study and the processes and methodologies used to collect the data.

\section{Statistical analysis}

Numerical variables were expressed as the mean \pm standard deviation (SD) or as medians (Q1-Q3 quartiles), according to the data distribution. Categorical variables were expressed as numbers (percentage). Differences in numerical variables between groups were analyzed by the independent $t$ test, analysis of variance (ANOVA), the Mann-Whitney $U$ test or the Kruskal-Wallis $H$ test, as appropriate, and differences in categorical variables were analyzed by the chi-square test. Multiple linear regression was used to estimate the associations between coronary lesion severity and levels of serum lipid parameters and hs-CRP. Cox proportional hazard regression analysis was performed to examine the association between MACE and levels of serum lipid parameters and hs-CRP. TG and hs-CRP levels were log-transformed in 
the multiple linear regression and Cox regression analysis due to the positively skewed nature of the distribution. SPSS software (version 20.0; SPSS Inc., Cary, Chicago, USA) was used to perform the statistical analyses. For all analyses, two-tailed $p$ values $<0.05$ were considered statistically significant.

\section{Results}

\section{Baseline characteristics}

A total of 4090 patients with stable CAD were enrolled in this study. As shown in Table 1, they were categorized into tertile subgroups according to their GS. There were significant differences in the levels of TC, LDL-C, HDL-C, TG and hs-CRP among the three subgroups (all $p<0.01$ ). The subgroups also differed significantly with respect to age, the percentage of male patients, systolic pressure, smoking status, hypertension and diabetes history, and fasting glucose and HbA1c levels (all $p<0.01$ ).

\section{Coronary lesion severity}

We evaluated the association between GS and metabolic risk factors using multiple linear regression analysis (Table 2). We found that LDL-C and hs-CRP were positively associated with GS (both $p<0.001$ ). By contrast,
HDL-C was negatively associated with GS $(\mathrm{p}<0.001)$. However, there were no associations between GS and levels of TC and TG in this study. Age, male gender, hypertension, diabetes and current smoking history were all positively associated with the GS (all $p<0.05$ ) (Table 2).

\section{Levels of serum lipids and hs-CRP after 12-week OMT}

We measured the levels of lipid parameters and hs-CRP of participants after 12-week OMT. There were significant decreases in TC, LDL-C, TG and hs-CRP levels, and increases in HDL-C levels (All $\mathrm{p}<0.05$ ). It should be noted that $91.2 \%(3730 / 4090)$ patients achieved the therapeutic goal for LDL-C $(<1.8 \mathrm{mmoL} / \mathrm{L})$.

\section{Mace}

We found that $471(11.5 \%)$ patients experienced MACE within a mean follow-up period of 39.5 months. Among them, $56(1.4 \%)$ patients suffered cardiovascular deaths, 138 (3.4\%) patients suffered non-fatal myocardial infarctions, $34(0.8 \%)$ patients suffered non-fatal strokes, and $243(5.9 \%)$ patients underwent unplanned coronary revascularization.

Table 1 Baseline characteristics of the study population according to Gensini score tertiles

\begin{tabular}{|c|c|c|c|c|c|}
\hline & \multirow{3}{*}{$\begin{array}{l}\text { Overall } \\
(n=4090)\end{array}$} & \multicolumn{3}{|c|}{ Gensini score category } & \multirow[t]{3}{*}{$p$} \\
\hline & & $<26$ & $26-43$ & $\geq 44$ & \\
\hline & & $(n=1309)$ & $(n=1405)$ & $(n=1376)$ & \\
\hline \multicolumn{6}{|l|}{ Clinical characteristics } \\
\hline Age, years & $61.1 \pm 10.9$ & $58.9 \pm 10.8$ & $61.8 \pm 10.7$ & $62.6 \pm 10.8$ & $<0.001$ \\
\hline Gender (male), \% (n) & $67.9(2779)$ & $62.9(823)$ & $68.4(961)$ & $72.3(995)$ & $<0.001$ \\
\hline $\mathrm{BMl}, \mathrm{kg} / \mathrm{m}^{2}$ & $25.0 \pm 3.2$ & $25.0 \pm 3.3$ & $25.0 \pm 3.1$ & $25.1 \pm 3.1$ & 0.262 \\
\hline Systolic pressure, mm Hg & $134 \pm 20$ & $132 \pm 19$ & $134 \pm 20$ & $135 \pm 21$ & 0.002 \\
\hline Diastolic pressure, mm Hg & $78 \pm 12$ & $78 \pm 12$ & $78 \pm 12$ & $78 \pm 13$ & 0.726 \\
\hline Hypertension, \% (n) & $57.8(2362)$ & $52.6(689)$ & $58.9(827)$ & $61.5(846)$ & $<0.001$ \\
\hline Diabetes mellitus, \% (n) & 23.9(977) & $22.2(290)$ & $22.4(315)$ & $27.0(372)$ & 0.003 \\
\hline Current smoking, \% (n) & 35.2(1438) & $31.0(406)$ & $34.2(480)$ & $40.1(552)$ & $<0.001$ \\
\hline Family history of premature CAD, \% (n) & $145(3.5)$ & $45(3.4)$ & $60(4.3)$ & $40(2.9)$ & 0.146 \\
\hline LVEF, \% & $61.1 \pm 6.9$ & $61.3 \pm 7.0$ & $61.0 \pm 6.9$ & $61.0 \pm 6.8$ & 0.594 \\
\hline \multicolumn{6}{|l|}{ Biochemistry parameters } \\
\hline $\mathrm{TC}, \mathrm{mmol} / \mathrm{L}$ & $4.64 \pm 1.08$ & $4.56 \pm 1.02$ & $4.61 \pm 1.05$ & $4.74 \pm 1.16$ & $<0.001$ \\
\hline $\mathrm{LDL}-\mathrm{C}, \mathrm{mmol} / \mathrm{L}$ & $2.78 \pm 0.91$ & $2.68 \pm 0.84$ & $2.74 \pm 0.90$ & $2.91 \pm 0.96$ & $<0.001$ \\
\hline $\mathrm{HDL}-\mathrm{C}, \mathrm{mmol} / \mathrm{L}$ & $1.14 \pm 0.31$ & $1.18 \pm 0.32$ & $1.12 \pm 0.31$ & $1.12 \pm 0.30$ & $<0.001$ \\
\hline $\mathrm{TG}, \mathrm{mmol} / \mathrm{L}$ & $1.51(1.07-2.18)$ & $1.48(1.06-2.12)$ & $1.46(1.05-2.10)$ & $1.57(1.12-2.28)$ & 0.003 \\
\hline $\mathrm{Hs}-\mathrm{CRP}, \mathrm{mg} / \mathrm{L}$ & $2.63(1.10-7.10)$ & $2.47(1.00-6.37)$ & $2.41(1.06-6.66)$ & $2.99(1.23-8.39)$ & $<0.001$ \\
\hline Fasting glucose, $\mathrm{mmol} / \mathrm{L}$ & $6.26 \pm 2.39$ & $6.00 \pm 2.11$ & $6.27 \pm 2.37$ & $6.50 \pm 2.62$ & $<0.001$ \\
\hline $\mathrm{HbA1C},(\%)$ & $6.4 \pm 1.4$ & $6.3 \pm 1.4$ & $6.4 \pm 1.4$ & $6.6 \pm 1.5$ & $<0.001$ \\
\hline
\end{tabular}

Data are shown as mean \pm standard deviation, median (Q1-Q3 quartiles), or percentages $(\mathrm{n})$. $P$ values from analysis of the variance (ANOVA), Kruskal-Wallis $\mathrm{H}$ tests, or chi-square tests. Two-tailed $p<0.05$ was considered statistically significant. CAD coronary artery disease, $B M I$ body mass index, $L V E F$ left ventricular ejection fraction, TC total cholesterol, LDL-C LDL cholesterol, HDL-C HDL cholesterol, TG triglyceride, $h s$-CRP high-sensitivity C reactive protein, HbA1C Hemoglobin A1c 
Table 2 Multiple linear regression analysis for the association of metabolic risk factors with coronary severity

\begin{tabular}{lll}
\hline Variables & Standardized coefficients & $p$ \\
\hline Age & 0.188 & $<0.001$ \\
Gender (male vs. female) & 0.082 & $<0.001$ \\
BMI & -0.001 & 0.963 \\
History of hypertension & 0.033 & 0.031 \\
(with vs. without) & & \\
History of diabetes mellitus & 0.048 & 0.002 \\
(with vs. without) & & $<0.001$ \\
History of current smoking & 0.088 & \\
(with vs. without) & & 0.537 \\
Family history of premature CAD & -0.009 & 0.267 \\
TC & -0.045 & $<0.001$ \\
LDL-C & 0.161 & $<0.001$ \\
HDL-C & -0.077 & 0.185 \\
TG (log-transformed) & 0.028 & $<0.001$ \\
Hs-CRP (log-transformed) & 0.166 &
\end{tabular}

$P$ values were from linear regression. Two-tailed $p<0.05$ was considered statistically significant. $C A D$ coronary artery disease, $B M I$ body mass index, $T C$ total cholesterol, LDL-C LDL cholesterol, HDL-C HDL cholesterol, TG triglyceride, hs-CRP high-sensitivity $C$ reactive protein

To determine whether baseline levels of hs-CRP, HDL-C and TG were associated with MACE, we performed Cox proportional hazard regression analysis. In the univariate analysis (Table 3), hs-CRP was associated with MACE, while HDL-C and TG were not. Furthermore, in the multivariate analysis adjusting for traditional risk factors, we found that there was a significant

Table 3 Univariate Cox regression analysis for the predictors of major adverse cardiovascular event

\begin{tabular}{llll}
\hline Variables & $H R$ & $95 \% \mathrm{Cl}$ & $p$ \\
\hline Age & 0.99 & $0.99-1.00$ & 0.117 \\
Gender (male vs. female) & 1.05 & $0.87-1.28$ & 0.596 \\
BMI & 1.02 & $0.99-1.05$ & 0.141 \\
History of hypertension (with vs. without) & 1.10 & $0.91-1.32$ & 0.321 \\
History of diabetes mellitus (with vs. without) & 1.64 & $1.35-1.99$ & $<0.001$ \\
History of current smoking (with vs. without) & 1.15 & $0.95-1.38$ & 0.153 \\
Family history of premature CAD & 1.22 & $0.78-1.92$ & 0.376 \\
Coronary revascularization & 0.82 & $0.68-0.98$ & 0.028 \\
LVEF, \% & 1.00 & $0.99-1.01$ & 0.851 \\
TC & 1.25 & $1.16-1.35$ & $<0.001$ \\
LDL-C & 1.37 & $1.27-1.48$ & $<0.001$ \\
HDL-C & 1.15 & $0.86-1.53$ & 0.344 \\
TG (log-transformed) & 1.07 & $0.92-1.25$ & 0.363 \\
Hs-CRP (log-transformed) & 1.19 & $1.09-1.30$ & $<0.001$ \\
\hline P &
\end{tabular}

$P$ values were from Cox proportional hazard regression. Two-tailed $p<0.05$ was considered statistically significant. $C A D$ Coronary artery disease, $B M I$ body mass index, TC total cholesterol, LDL-C LDL cholesterol, HDL-C HDL cholesterol, $T G$ triglyceride, $h s-C R P$ high-sensitivity $C$ reactive protein, $H R$ hazard ratio, $\mathrm{Cl}$ confidence interval difference in the adjusted event-free survival rate among the hs-CRP quartile subgroups $(p=0.001)$ (Fig. 2). The hazard ratio for MACE was 1.17 (95\% confidence interval: $1.07-1.28, p<0.001$ ) per 3.9 -fold increase in the hs-CRP concentration [i.e., per one standardized deviation increase in the log-transformed hs-CRP level] (Table 4). The LDL-C was also associated with MACE $(\mathrm{p}<0.001)$. However, HDL-C and TG levels were not predictors of MACE. We also noticed that diabetes was positively and coronary revascularization was negatively associated with MACE (both $p<0.05$ ).

\section{Discussion}

In this prospective study, we found that, baseline hs-CRP level was an independent predictor of MACE in a cohort of Chinese population with stable CAD who received OMT within a mean follow-up period of 39.5 months. However, HDL-C and TG levels were not associated with MACE. Our findings can serve as new evidences for the contribution of inflammation to residual cardiovascular risk in Chinese population.

Residual cardiovascular risk in the setting of adequately controlled LDL-C has been a major concern for the treatment of CAD. In this study, 11.5\% (471/4090) of patients, suffered MACE despite receiving OMT during an average of 39.5 months fellow-up period. It should be noted that $91.2 \%$ (3730/4090) patients had achieved the therapeutic goal for LDL-C $(<1.8 \mathrm{mmol} / \mathrm{L})$ after 3 month OMT. The finding was similar to those of our previous studies in Chinese population [22, 23].

LDL-C is a well- established risk factor of CAD. It has been shown that lowering of circulated LDL-C dose dependently results in reduction of cardiovascular events. Every $1 \mathrm{mmol} / \mathrm{L}$ reduction in LDL-C is associated with a corresponding $22 \%$ decrease in CAD mortality and morbidity [2]. Consistently, we found that baseline LDL-C was associated with MACE in this study.

Substantial experimental work has elucidated molecular and cellular pathways of inflammation that promote atherosclerosis [24]. Evidences from epidemiologic studies indicated that several inflammatory makers, including hs-CRP, interleukin-6 (IL-6), tumor necrosis factor- $\alpha$ (TNF- $\alpha$ ), etc., were positively associated with cardiovascular risk in western population [25-27]. Also, patients with insulin resistance, regarded as a chronic inflammatory event, had increased risk of cardiovascular events $[28,29]$. Contrarily, some molecules that are known to have anti-inflammatory action, e.g. Ghrelin, have shown some beneficial effects on cardiovascular system [30]. Of which, hs-CRP is the most well-recognized and widely-used inflammatory marker for cardiovascular risk assessment. Paul et al. found that, of the 12 markers measured, hs-CRP was the strongest univariate predictor of the risk of cardiovascular events [25]. More 
A

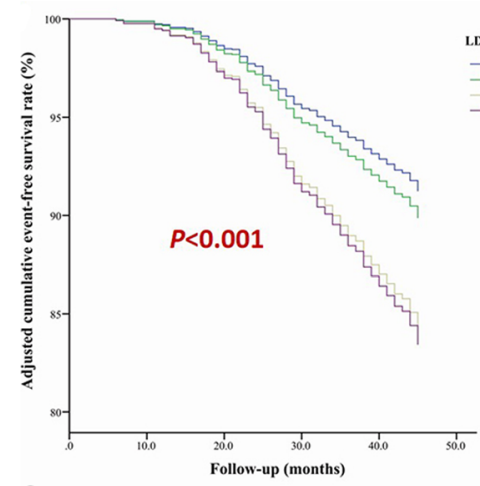

C

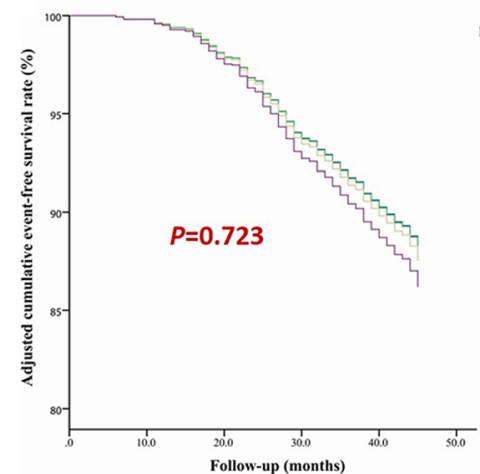

B

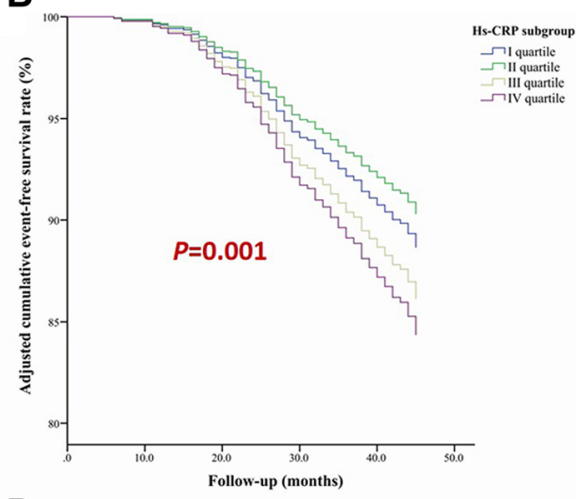

D

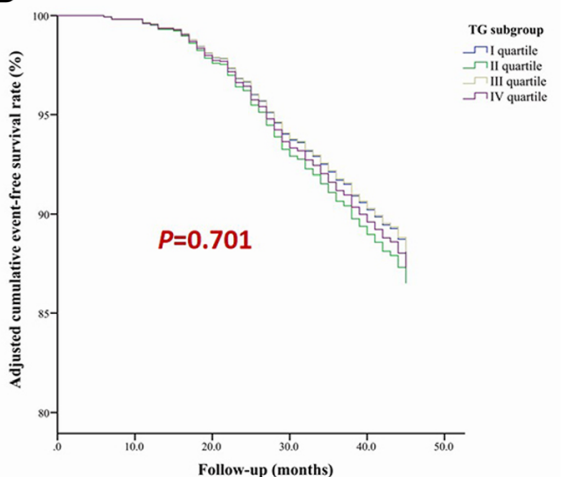

Fig. 2 The adjusted cumulative event-free survival rate of the study population. There existed significant differences in the adjusted cumulative event-free survival rate among LDL-C and hs-CRP subgroups ( $p=0.001)$.LDL-C: low density lipoprotein cholesterol, hs-CRP: high sensitivity $C$ reactive protein; HDL-C: high density lipoprotein cholesterol; TG: triglyceride

Table 4 Multivariate Cox regression analysis for the independent predictors of major adverse cardiovascular event

\begin{tabular}{llll}
\hline Variables & $H R$ & $95 \% \mathrm{Cl}$ & $p$ \\
\hline Age & 1.00 & $0.99-1.01$ & 0.600 \\
Gender (male vs. female) & 1.09 & $0.86-1.36$ & 0.483 \\
BMI & 1.00 & $0.97-1.03$ & 0.754 \\
History of hypertension (with vs. without) & 1.09 & $0.90-1.32$ & 0.366 \\
History of diabetes mellitus (with vs. without) & 1.69 & $1.39-2.05$ & $<0.001$ \\
History of current smoking (with vs. without) & 1.06 & $0.85-1.32$ & 0.595 \\
Family history of premature CAD & 1.20 & $0.77-1.89$ & 0.420 \\
Coronary revascularization & 0.82 & $0.68-0.98$ & 0.027 \\
LVEF, \% $_{\text {TC }}{ }^{\text {a }}$ & 1.00 & $0.99-1.01$ & 0.770 \\
LDL-C $^{a}$ & 0.78 & $0.60-1.01$ & 0.056 \\
HDL-C $^{\text {a }}$ & 1.63 & $1.30-2.05$ & $<0.001$ \\
TG $^{\mathrm{a}}$ (log-transformed) & 1.10 & $0.97-1.24$ & 0.125 \\
Hs-CRP $^{\text {a }}$ (log-transformed) & 1.06 & $0.93-1.21$ & 0.375 \\
\hline
\end{tabular}

$P$ values were from Cox proportional hazard regression. Two-tailed $p<0.05$ was considered statistically significant. $C A D$ Coronary artery disease, $B M I$ body mass index, TC total cholesterol, LDL-C LDL cholesterol, HDL-C HDL cholesterol, $T G$ triglyceride, $h s-C R P$ high-sensitivity $C$ reactive protein, $H R$ hazard ratio, $C l$ confidence interval. ${ }^{a}$ One standard deviation increase importantly, it has been recently reported that pharmacological inhibition of inflammation with canakinumab reduced cardiovascular events in CAD patients with hs-CRP levels of more than $2 \mathrm{mg} / \mathrm{L}$ [5]. Thus, the newly released clinical practice guideline from American College of Cardiology/American Heart Association recommends elevated hs-CRP level $(\geq 2 \mathrm{mg} / \mathrm{L})$ as a risk-enhancing factor when assessing cardiovascular risk [31]. Our study indicated that hs-CRP level was an independent predictor of residual risk of cardiovascular events in Chinese population. Future clinical trials can testify the benefits of anti-inflammation therapy in Chinese patients with CAD.

Nevertheless, we found that the baseline levels of TG and HDL-C were not associated with MACE. Elevated TG and decreased HDL-C are more frequently seen than high LDL-C level in Chinese population [32]. The role of TG and HDL-C in CAD still remain controversial. Although observational studies have suggested serum TG level is associated with CAD risk, randomized controlled trials of fibrates and omega-3 fatty acids to reduce TG failed to show any significant clinical benefits $[9,11]$. The fact that atherosclerotic plaques possess primarily cholesterol instead of TG also objects to the premise 
that TG is directly involved in plaque formation. However, recent genetic studies showed some variants in gene loci involved in plasma TG metabolism are associated with the risk of CAD [14-16]. Genetic evidence is free of confounders and reverse causation and is thus helpful for identifying causal risk factors for CAD. Therefore, renewed interests are gained on these gene loci and corresponding encoding products, such as apolipoprotein C3 $[15,16]$. Future research at this point will provide new insight to the understanding of the role of TG in CAD. HDL exerts various athero-protective properties, including mediating cholesterol efflux, protecting vascular endothelium, anti-inflammatory and anti-apoptotic effects [7, 33]. However, medication aimed to increase HDL-C levels didn't reduce CAD risk $[8,10,12,13]$. Genetic studies also didn't support HDL-C as a risk factor of CAD [7]. Recent studies suggested that it is HDL function but not HDL-C levels that play a role in CAD development [7].

Due to the observational nature of this study, we could not determine the benefits of treatment to reduce hs-CRP or TG, or increase HDL-C levels in Chinese population. Future clinical trials will provide more comprehensive evidences.

\section{Conclusions}

In summary, our study demonstrated that, baseline hs-CRP levels was an independent risk factor for MACE in a cohort of Chinese population with stable CAD who received OMT. However, TG and HDL-C levels were not associated with MACE.

\begin{abstract}
Abbreviations
ANOVA: analysis of variance; CABG: coronary artery bypass grafting; CAD: coronary artery disease; CCB: calcium channel-blocking; GS: Gensini score; HbA1c: Hemoglobin A1c; HDL-C: high-density lipoprotein cholesterol; hs-CRP: high sensitivity C reactive protein; IL-6: interleukin-6; IL- $\beta$ : interleukin$1 \beta$; LDL-C: low density lipoprotein cholesterol; LVEF: left ventricular ejection fraction; MACE: major adverse cardiovascular event:" OMT: optimal medical treatment; $\mathrm{PCl}$ : percutaneous coronary intervention; SD: standard deviation; TC: total cholesterol; TG: triglyceride; TNF-a: tumor necrosis factor-a; $\beta$ blockers: beta-receptor blockers
\end{abstract}

\section{Acknowledgements \\ We thank investigators as follows: Fang Wang, Department of Cardiology, Beijing Hospital; Jianxiong Liu, Department of Cardiology, Chengdu Second People's Hospital; Dingli Xu, Department of Cardiology, Nanfang Hospital, Southern Medical University; Changgian Wang, Shanghai Ninth People's Hospital, Shanghai Jiaotong University; Yujie Zhou, Department of Cardiology, Beijing Anzhen Hospital, Capital Medical University; Guosheng Fu, Department of Cardiology, Shaoyifu Hospital, Zhejiang University; Luosha Zhao, Yu Xing, Department of Cardiology, The First Affiliated Hospital of Zhengzhou University; Daqing Zhang, Department of Cardiology, Shengjing Hospital of China Medical University; Biao Cheng, Department of Cardiology, People's Hospital of Sichuan Province; Yi An, Department of Cardiology, Cardiovascular Hospital of Qingdao University; Dong Xu, Department of Cardiology, Xuanwu Hospital, Capital Medical University; Ling Han, Department of Cardiology, Fuxing Hospital, Capital Medical University.}

\section{Funding}

National Natural Science Foundation of China (81170262).

\section{Availability of data and materials}

The datasets used and/or analysed during the current study are available from the corresponding author on reasonable request.

\section{Authors' contributions}

Conceived and designed the study: SZ. Performed the study: WD and ZZ. Analyzed the data: WD and ZZ. Wrote the paper: WD and SZ. All authors read and approved the final manuscript.

\section{Ethics approval and consent to participate}

This study was approved by the ethics committee review board of The Second Xiangya Hospital of Central South University. Informed written consent was obtained from all participants.

\section{Consent for publication}

Not applicable.

\section{Competing interests}

The authors declare that they have no competing interests.

\section{Publisher's Note}

Springer Nature remains neutral with regard to jurisdictional claims in published maps and institutional affiliations.

Received: 29 August 2018 Accepted: 21 November 2018

Published online: 03 December 2018

\section{References}

1. Jacobson $T A$, Ito MK, Maki KC, Orringer $C E$, Bays $H E$, Jones $P H$, et al. National Lipid Association recommendations for patient-centered management of dyslipidemia: part 1 - executive summary. J Clin Lipidol. 2014;8(5):473-88.

2. Ridker PM. LDL cholesterol: controversies and future therapeutic directions. Lancet. 2014:384(9943):607-17.

3. Cholesterol Treatment Trialists' (CTT) Collaboration, Baigent C, Blackwell L, Emberson J, Holland LE, Reith C, Bhala N, Peto R, Barnes EH, Keech A, Simes J, Collins R. Efficacy and safety of more intensive lowering of LDL cholesterol: a meta-analysis of data from 170000 participants in 26 randomised trials. Lancet. 2010;376(9753):1670-81.

4. Farnier M. Future lipid-altering therapeutic options targeting residual cardiovascular risk. Curr Cardiol Rep. 2016;18(7):65.

5. Ridker PM, Everett BM, Thuren T, MacFadyen JG, Chang WH, Ballantyne C, et al. Antiinflammatory Therapy with Canakinumab for Atherosclerotic Disease. N Engl J Med. 2017;377(12):1119-31.

6. Nordestgaard BG, Varbo A. Triglycerides and cardiovascular disease. Lancet. 2014;384:626-35.

7. Rader DJ, Hovingh GK. HDL and cardiovascular disease. Lancet. 2014;384: 618-25.

8. Barter PJ, Caulfield M, Eriksson M, Grundy SM, Kastelein JJ, Komajda M, et al. Effects of torcetrapib in patients at high risk for coronary events. N Engl J Med. 2007:357(21):2109-22.

9. Group AS, Ginsberg HN, Elam MB, Lovato LC, Crouse JR 3rd, Leiter LA, et al. Effects of combination lipid therapy in type 2 diabetes mellitus. N Engl J Med. 2010;362(17):1563-74.

10. Investigators A-H, Boden WE, Probstfield JL, Anderson T, Chaitman BR, Desvignes-Nickens P, et al. Niacin in patients with low HDL cholesterol levels receiving intensive statin therapy. N Engl J Med. 2011;365(24):2255-67.

11. Investigators OT, Bosch J, Gerstein HC, Dagenais GR, Diaz R, Dyal L, et al. N-3 fatty acids and cardiovascular outcomes in patients with dysglycemia. N Engl J Med. 2012;367(4):309-18.

12. Lincoff AM, Nicholls SJ, Riesmeyer JS, Barter PJ, Brewer HB, Fox KAA, et al. Evacetrapib and cardiovascular outcomes in high-risk vascular disease. N Engl J Med. 2017;376(20):1933-42.

13. Group HTC. HPS2-THRIVE randomized placebo-controlled trial in 25673 high-risk patients of ER niacin/laropiprant: trial design, pre-specified muscle and liver outcomes, and reasons for stopping study treatment. Eur Heart J. 2013:34(17):1279-91.

14. Do R, Willer CJ, Schmidt EM, Sengupta S, Gao C, Peloso GM, et al. Common variants associated with plasma triglycerides and risk for coronary artery disease. Nat Genet. 2013;45:1345-52. 
15. Crosby J, Peloso GM, Auer PL, Crosslin DR, Stitziel NO, Lange LA, et al. Lossof-function mutations in APOC3, triglycerides, and coronary disease. N Engl J Med. 2014;371(1):22-31.

16. Jorgensen AB, Frikke-Schmidt R, Nordestgaard BG, Tybjaerg-Hansen A. Lossof-function mutations in $\mathrm{APOC} 3$ and risk of ischemic vascular disease. N Engl J Med. 2014;371(1):32-41.

17. Dai W, Long J, Cheng Y, Chen Y, Zhao S. Elevated plasma lipoprotein (a) levels were associated with increased risk of cardiovascular events in Chinese patients with stable coronary artery disease. Sci Rep. 2018;8(1):7726.

18. Task Force M, Montalescot G, Sechtem U, Achenbach S, Andreotti F, Arden C, et al. 2013 ESC guidelines on the management of stable coronary artery disease: the task force on the management of stable coronary artery disease of the European Society of Cardiology. Eur Heart J. 2013;34(38):2949-3003.

19. Qaseem A, Finn SD, Williams S, Dallas P, Owens DK, Shekelle P. Diagnosis of stable ischemic heart disease: summary of a clinical practice guideline from the American College of Physicians/American College of Cardiology Foundation/American Heart Association/American Association for Thoracic Surgery/preventive cardiovascular nurses association/Society of Thoracic Surgeons. Ann Intern Med. 2012;157(10):729-34.

20. Gensini GG. A more meaningful scoring system for determining the severity of coronary heart disease. Am J Cardiol. 1983;51(3):606.

21. Zhao S, Wang Y, Mu Y, Yu B, Ye P, Yan X, et al. Prevalence of dyslipidaemia in patients treated with lipid-lowering agents in China: results of the DYSlipidemia international study (DYSIS). Atherosclerosis. 2014;235(2):463-9.

22. Zhao SP, Yu BL, Peng DQ, Huo Y. The effect of moderate-dose versus double-dose statins on patients with acute coronary syndrome in China: results of the CHILLAS trial. Atherosclerosis. 2014;233(2):707-12.

23. Lu Z, Kou W, Du B, Wu Y, Zhao S, Brusco OA, et al. Effect of Xuezhikang, an extract from red yeast Chinese rice, on coronary events in a Chinese population with previous myocardial infarction. Am J Cardiol. 2008;101(12): 1689-93.

24. Libby P. Inflammation in atherosclerosis. Arterioscler Thromb Vasc Biol. 2012; 32(9):2045-51.

25. Ridker PM, Hennekens CH, Buring JE, Rifai N. C-reactive protein and other markers of inflammation in the prediction of cardiovascular disease in women. N Engl J Med. 2000;342(12):836-43.

26. Hansson GK. Inflammation, atherosclerosis, and coronary artery disease. N Engl J Med. 2005;352(16):1685-95

27. Ridker PM, Cannon CP, Morrow D, Rifai N, Rose LM, McCabe CH, et al. Creactive protein levels and outcomes after statin therapy. N Engl J Med. 2005;352(1):20-8.

28. Bornfeldt KE, Tabas I. Insulin resistance, hyperglycemia, and atherosclerosis. Cell Metab. 2011;14(5):575-85.

29. Patel TP, Rawal K, Bagchi AK, Akolkar G, Bernardes N, Dias Dda S, et al. Insulin resistance: an additional risk factor in the pathogenesis of cardiovascular disease in type 2 diabetes. Heart Fail Rev. 2016;21(1):11-23.

30. Lilleness BM, Frishman WH. Ghrelin and the cardiovascular system. Cardiol Rev. 2016;24(6):288-97.

31. Grundy SM, Stone NJ, Bailey AL, Beam C, Birtcher KK, Blumenthal RS, et al. 2018 AHA/ACC/AACVPR/AAPA/ABC/ACPM/ADA/AGS/APhA/ASPC/NLA/ PCNA guideline on the Management of Blood Cholesterol. A Report of the American College of Cardiology/American Heart Association Task Force on Clinical Practice Guidelines 2018.

32. Yang W, Xiao J, Yang Z, Ji L, Jia W, Weng J, et al. Serum lipids and lipoproteins in Chinese men and women. Circulation. 2012;125(18):2212-21.

33. Rosenson RS, Brewer HB Jr, Ansell B, Barter P, Chapman MJ, Heinecke JW, et al. Translation of high-density lipoprotein function into clinical practice: current prospects and future challenges. Circulation. 2013;128(11):1256-67.

\section{Ready to submit your research? Choose BMC and benefit from:}

- fast, convenient online submission

- thorough peer review by experienced researchers in your field

- rapid publication on acceptance

- support for research data, including large and complex data types

- gold Open Access which fosters wider collaboration and increased citations

- maximum visibility for your research: over $100 \mathrm{M}$ website views per year

At $\mathrm{BMC}$, research is always in progress.

Learn more biomedcentral.com/submissions 